\title{
Kulturelles ERBE
}

Prof. Dr. jur. Ellen Euler, LL.M."

\section{Next Level Hack: Aktuelle Rechtsprechung zum Framing und deren Auswirkungen auf den Zugang zu Kultur im Internet}

Framing war wiederholt Gegenstand höchstrichterlicher Rechtsprechung und wissenschaftlicher Auseinandersetzungen in Deutschland und Europa. Der vorliegende Beitrag nimmt eine Entscheidung des Europäischen Gerichtshofs (EuGH) von Anfang März 2021 für eine Einordnung und das Aufzeigen der möglichen kulturpolitischen Konsequenzen zum Anlass. ${ }^{1}$

\section{Problemaufriss}

Einleitend soll neben der Präzisierung der Begrifflichkeiten kurz die rechtliche Problematik auf der Grundlage der Entwicklung der Rechtsprechung des EuGH zum Framing aufgezeigt werden.

\section{Framing / Linking \& Embedding - eine begriffliche Einordnung}

Als Framing wird in Rechtsprechung und Literatur ein Link bezeichnet, der Inhalte oder Code von einem fremden Webserver in die eigene Website einbettet. Abhängig von der jeweiligen Technik ist es für Nutzer\%innen nicht unmittelbar ersichtlich, dass der Inhalt der aufgerufenen Website nicht unmittelbar durch eigenen Upload zur Verfügung gestellt wird, sondern teilweise ein fremdes Angebot dem eigenen hinzufügt. ${ }^{2}$ Die Bezeichnung stammt von dem HTML-Element „frame“, das den Webbrowser des Nutzers anweist, Inhalte der angegebenen URL (z.B. einen Deep-Link auf einen

* Die Verfasserin ist Professorin an der Fachhochschule Potsdam am Fachbereich Informationswissenschaften und lehrt dort mit Schwerpunkt Open Access/Open Data/Open Science.

1 EuGH C-329/19, Urt. v. 09.03.2021 - ECLI:EU:C:2020:696 - VG Bild-Kunst, abrufbar unter: https://t1p.de/vh5m, zuletzt abgerufen am 11.05.2021.

$2 \mathrm{Zu}$ technischen Einzelheiten siehe Conrad, Kuck mal wer da spricht; in: Computer \& Recht (CR) 2013, 305. abrufbar unter: https://papers.ssrn.com/sol3/papers.cfm?abstract_id=224987 9, zuletzt abgerufen am 18.04.2021. 
anderen Server) zu laden und in einem Rahmen (Frame) darzustellen. ${ }^{3}$ Diese Technik nutzt die Funktionalität eines Hyperlinks, d.h., das digitale Objekt, z.B. ein Bild, wird im Browser von seinem ursprünglichen Platz aus automatisch auf der einbindenden Website angezeigt. Aus der Sicht der Nutzer*in entspricht die Wirkung der des eigenen Uploads. Diese Praxis ist als Inline Linking oder Hotlinking bekannt. Framing wird heute als obsolet angesehen und ist in der bisher letzten Fassung der Auszeichnungssprache HTML (HTML5) nicht mehr vorgesehen. An seine Stelle ist der Inlineframe getreten. Der Inlineframe verhält sich wie ein integrales Element dieser Seite, denn es handelt sich anders als beim klassischen Framing nicht um eine Technik der Bildschirmteilung, sondern um eine solche der Einbettung (embedding) externer Ressourcen in eine Webseite. ${ }^{4}$ Seither kann technologisch eigentlich nicht mehr von Framing gesprochen werden. Außer in der differenzierten Antwort im Schlussantrag des Generalanwalts des EuGHs im vorliegenden Fall, ${ }^{5}$ verwendet die Rechtsprechung pauschal den Begriff Framing für das Einbetten fremder Inhalte durch Dritte und differenziert insbesondere nicht zwischen iframes, bei denen noch ein Link aktiviert werden muss, bevor der Inhalt angezeigt wird, und solchen iframes, innerhalb derer ein externer Inhalt automatisch angezeigt beziehungsweise abgespielt wird. Entgegen der Empfehlung des Generalanwalts des EuGH in seinem Schlussantrag vom 10.09.2020, im Hinblick auf die rechtliche Bewertung zwischen den unterschiedlichen LinkingTechnologien zu differenzieren, nimmt der EuGH in seiner Entscheidung vom 09.03.2021 weiterhin eine pauschale Betrachtung des Framing vor und unterscheidet nicht zwischen den unterschiedlichen Linking-Technologien.

\section{Rechtliche Problematik \& Beurteilung}

Immer wieder war auf nationaler Ebene streitig, ob und wenn ja in welchem Umfang das Verlinken von Inhalten Dritter im Internet als öffentliche Zugänglichmachung im Sinne des $\mathbb{1} 15$ Abs. 2 Nr. 2 S. 2 i.V.m $\mathbb{1 9 a}$ UrhG, oder als unbenannte Form der öffentlichen Wiedergabe im Sinne des $₫ 15$ Abs. 2 S. 1 UrhG und damit als urheberrechtliche Nutzungshandlung einzuordnen ist. ${ }^{6}$

3 Lotze/Heinson in: Hasselblatt, MAH Gewerblicher Rechtsschutz, München 5. Aufl. 2017, Rdn. 96.

4 Siehe: https://www.w3.org/TR/html5-diff/\#absent-elements, zuletzt abgerufen am 11.05.2021, zum html-tag iframe: https://www.w3schools.com/tags/tag_iframe.as, zuletzt abgerufen am 11.05.2021.

5 Schlussantrag des Generalanwalts in der Rechtssache C-329/19 v. 10.09.2020, abrufbar unter: https://t1p.de/wvpc, zuletzt abgerufen am 11.05.2021.

6 BGH, Urt. v. 29.04.2010 - I ZR 39/08 - Session-ID, abrufbar unter: https://t1p.de/hri4, zuletzt abgerufen am 11.05.2021; BGH, Urt. v. 16.05.2013 - I ZR 46/12 - Die Realität I, abrufbar unter: https://t1p.de/bm2y, zuletzt abgerufen am 11.05.2021; BGH, Urt. v. 09.07.2015 - I ZR 46/12 - Die Realität II, abrufbar unter: https://t1p.de/qurd, zuletzt abgerufen am 11.05.2021; BGH, Urt. v. 29.4.2010 - I ZR 69/08 - Vorschaubilder I, abrufbar unter: https://t1p.de/d2mg, zuletzt abgerufen am 11.05.2021; BGH, Urt. v. 19.10.2011 - I ZR 140/10 - Vorschaubilder II, abrufbar unter: https://t1 p.de/b68d, zuletzt abgerufen am 11.05.2021; BGH, Urt. v. 21.09.2017 - I ZR 11/16 - Vorschaubilder III, abrufbar unter: https://t1p.de/bbml, zuletzt abgerufen am 
Vor dem Hintergrund der europarechtlichen Harmonisierung des Begriffes der öffentlichen Wiedergabe durch Art. 3 Abs. 1 und 2 der Richtlinie 2001/29 ist für die Beantwortung dieser Frage insbesondere die Rechtsprechung des EuGH zum Begriff der öffentlichen Wiedergabe zu berücksichtigen. ${ }^{7}$

Die jüngste Entscheidung geht auf eine jahrelange Vertragsverhandlung zwischen der Deutschen Digitalen Bibliothek (DDB) und der Verwertungsgesellschaft BildKunst e.V. (VG BK) zurück. Die DDB hatte diese im Jahr 2013 mit dem Ziel aufgenommen, eine Vereinbarung über die Lizenzierung des gesamten Portfolios geschützter visueller Werke zu treffen, 1. für den Upload von Vorschaubildern für die Zugänglichmachung auf Seiten der Kulturplattform DDB und 2. als Vertrag zugunsten Dritter auf Seiten der hieran beteiligten Kulturerbeeinrichtungen.

Während sich die Kulturplattformen in Deutschland und Europa im Bereich der textuellen Publikationen auf solche Materialien beschränken, die urheberrechtlich unproblematisch zugänglich gemacht werden können, weil Schutzrechte entweder abgelaufen sind, oder als verwaiste oder vergriffene Werke, oder weil im Wege des Open Access mit freien Lizenzen publiziert, wird im Bereich der visuellen Künste versucht, auch geschützte Werke einzubeziehen, um das schwarze Loch des 20 Jahrhundert im Hinblick auf die Verfügbarkeit von Kunst und Kultur auf Kulturplattformen ${ }^{8}$ in diesem Bereich zu verkleinern.

Damit soll die Sichtbarkeit kultureller Inhalte im Internet erleichtert und gefördert werden. Diese weitgehende Sichtbarkeit ist das von der DDB verfolgte Ziel. Die DDB macht als Plattform für Wissen und Kultur die Bestände aus den Wissensund Kultureinrichtungen in Deutschland verfügbar und aggregiert sie als nationaler Partner der europäischen Kulturplattform Europeana mit dem Ziel, das Wissen und die Kulturschätze aus Deutschland in Europa und der Welt sichtbar zu machen und zu vernetzen. Weitergehend soll so möglich werden, dass maschinelle Verfahren der Wissensgenerierung (z.B. quantitative Analyseverfahren wie das Text and Data Mining) und semantische Suchen möglich sind und das kulturelle Wissen in bisher unbekanntem Umfang kontextualisiert und angereichert sowie in Beziehung gesetzt

11.05.2021; BGH, Urt. v. 17.07.2003 - I ZR 259/00 - Paperboy, abrufbar unter: https://t1p.de/ spv3, zuletzt abgerufen am 11.05.2021.

7 EuGH, Urt. v. 21.10.2014 C-348/13 ECLI:EU:C:2014:2315 - BestWater International, abrufbar unter: https://t1p.de/66ms, zuletzt abgerufen am 11.05.2021; EuGH, Urt. v. 13.02.2014 ECLI:EU:C:2014:76 - C-466/12 Svensson, abrufbar unter: https://t1p.de/oogt, zuletzt abgerufen am 11.05.2021; EuGH, Urt. v. 08.09.2016 C-160/15 ECLI:EU:C:2016:644 - GS Media, abrufbar unter: https://t1p.de/8ch5, zuletzt abgerufen am 11.05.2021; EuGH, Urt. v. 07.08.2018 C-161/17 ECLI:EU:C:2018:634 - Renkhoff, abrufbar unter: https://t1p.de/hfhn, zuletzt abgerufen am 11.05.2021.

8 Welches dadurch entsteht, dass es aufgrund des engen europarechtlichen Rahmens auf nationaler Ebene keine „Katalogbildschranke“ geben kann, die die Kulturerbeeinrichtungen befähigt, ihre Bestände katalogisierend aufzuzeigen und zu vernetzen. Siehe auch: Euler/Dreier: „Onleihe und virtueller Museumsbummel. Das Menschenrecht auf kulturelle Teilhabe im 21. Jahrhundert“, in: Klimpel/Euler (Hrsg), Der Vergangenheit eine Zukunft - Kulturelles Erbe in der digitalen Welt, iRights Media: Berlin 2015, S. 192 ff., abrufbar unter: https://doi.org/10.171 76/20180716-114912-0. 
werden kann. Die umfassende Sichtbarkeit und Vernetzung sowie der Zugriff auf die kulturellen Wurzeln sollen kulturelle Fortschreibung und Innovation erleichtern und eine Standortbestimmung in Europa und der Welt auch digital ermöglichen. Die Sorge davor, dass der Kulturraum Europa im digitalen Raum durch die überrepräsentierten kulturellen Inhalte aus dem die Verfügbarmachung kultureller Inhalte im Internet weniger einschränkenden, angloamerikanischem Copyright-Raum überschrieben werden könnte, hat letzten Endes auch den Anstoß für die Förderung und Entwicklung von Kulturplattformen wie Europeana und DDB gegeben. ${ }^{9}$

Kurz vor Vertragsschluss Ende 2014 führten zwei neue Entscheidungen des EuGH zur öffentlichen Wiedergabe, die klar stellten, dass (einbettende) Links (außer mit Gewinnerzielungsabsicht) keine urheberrechtliche Nutzungshandlung (öffentliche Wiedergabe) bedeuten, weil damit kein neues Publikum adressiert wird, solange kein nenes technisches Verfahren zum Einsatz kommt, ${ }^{10}$ jedoch zu einem vorläufigen Stopp der Verhandlungen. Die VG BK verweigerte im Hinblick auf diese Rechtsprechung die Lizenzierung ohne die Implementierung von technischen Schutzmaßnahmen gegen das Framing Dritter, da diese aus Sicht der VG BK eine Enteignung der Bildurheber*innen bedeuten würde. ${ }^{11}$ Sie beklagt einen Kontrollverlust und Rechtlosstellung und fordert, dass Framing als urheberrechtlich relevante Nutzungshandlung im Sinne der öffentlichen Wiedergabe bewertet wird und daher ohne Einwilligung unzulässig ist. ${ }^{12}$ Diese Forderung korrespondiert mit dem Verlangen der Erweiterung der Verbietungsrechte und einer Regulierung des Framing auf europäischer und nationaler Ebene durch Korrektur der Rechtsprechung. ${ }^{13}$

9 Euler, Kultur \& Wissen online. Google darf und die Kulturerbeeinrichtungen? Blogbeitrag bei Deutsche Digitale Bibliothek 2016, abrufbar unter: https://doi.org/10.17176/20180718-1 54148-0.

10 Svensson und GS Media. Übersichtlich zusammengestellt ist die Frage, wann nach der Rechtsprechung des EuGH eine öffentliche Wiedergabe durch Verlinkung vorliegt, in der Tabelle auf S. 131.

11 Siehe VG Bild-Kunst: EuGH enteignet Bildurheber, abrufbar unter: https://perma.cc/T3LR -GUGC, zuletzt abgerufen am 11.05.2021.

12 Siehe stellvertretend die Justiziarin der VG Bild-Kunst Schierholz, Die Probleme der Rechtsprechung mit dem Framing - Anmerkung zu BGH, Urt. v. 21.9.2017 - I ZR 11/16 Vorschaubilder III, in: Zeitschrift für Urheber- und Medienrecht (ZUM) 2018, 135.

13 Siehe nur „Framing muss ins Brüsseler Paket“: https://web.archive.org/web/20161130223 040/http://www.bildkunst.de/urheberrecht/die-hyperlink-problematik/framing-muss-ins-b ruesseler-urheberrechts-paket.html, zuletzt abgerufen am 11.05.2021; „EuGH enteignet Urheber": https://web.archive.org/web/20161130170339/http://www.bildkunst.de/urheber recht/die-hyperlink-problematik/eugh-enteignet-bildurheber.html, zuletzt abgerufen am 11.05.2021; Position VG zur UrhReform EU: https://web.archive.org/web/2016113022 3216/http://www.bildkunst.de/urheberrecht/position-zur-geplanten-eu-reform-2016.html, zuletzt abgerufen am 11.05.2021 sowie zuletzt die formulierte Forderung an den EuGH klarzustellen, dass sich die Zustimmung zur Nutzung eines Werkes im Internet immer nur auf die konkrete Webseite bezieht und die Einbettung des Werkes in weiteren Webseiten nicht von einer solchen Zustimmung erfasst ist: https://perma.cc/T3LR-GUGC, zuletzt abgerufen am 11.05.2021. 


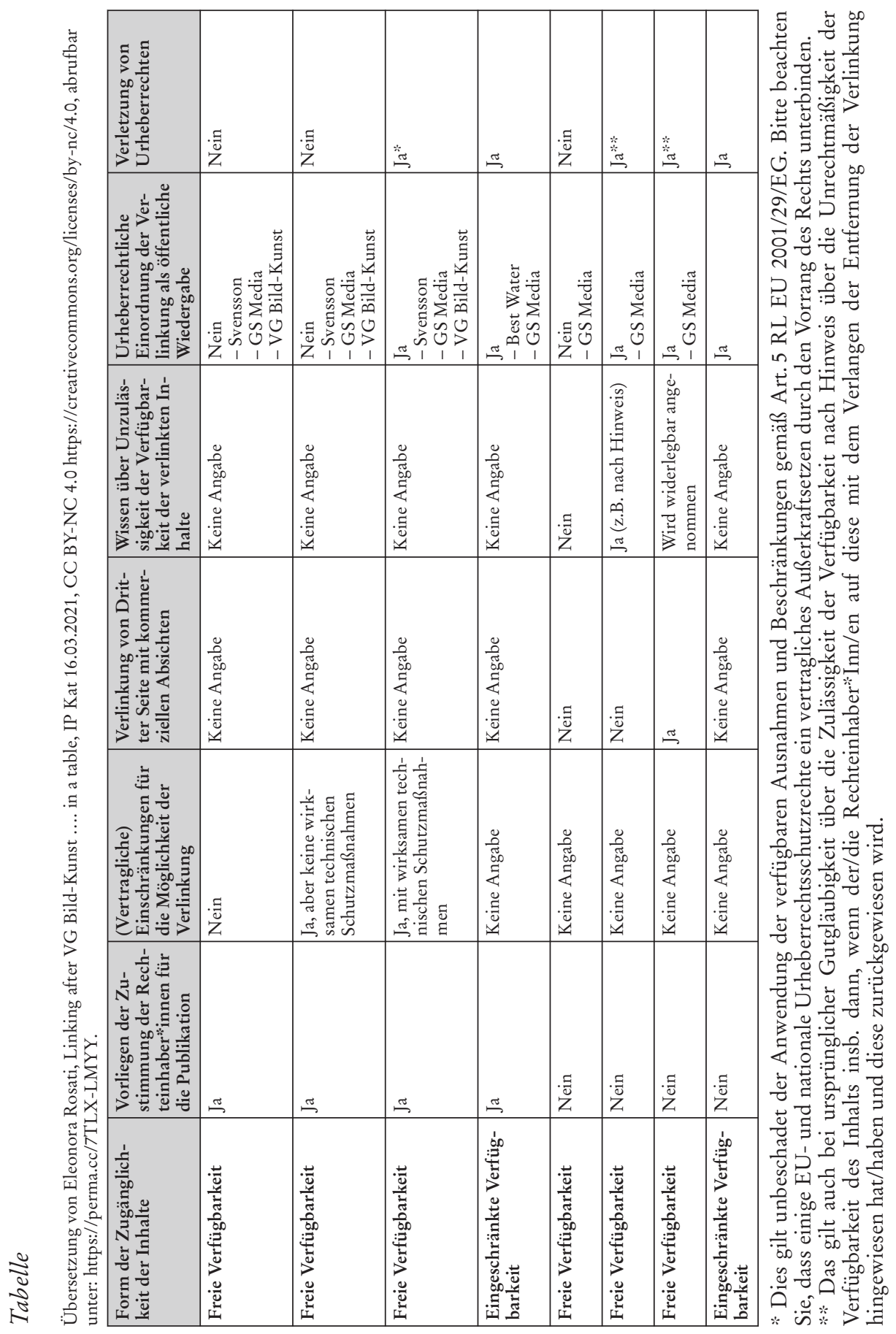

RuZ 2. Jg. 3/2021 
Auf EU-Ebene machten sich die EVA (European Visual Artists) ${ }^{14}$ der Internationale Verband der Bildagenturen, CEPIC ${ }^{15}$ und die Europäische Journalisten-Föderation (EFJ) für eine Korrektur der Rechtsprechung durch den europäischen Gesetzgeber stark. ${ }^{16}$

Statt einen Lizenzierungsvertrag zu schließen, einigten sich DDB und VG BK schließlich darauf, ein Musterverfahren zu führen, um die Frage zu klären, ob die Rechteinhaber*innen - vertreten über eine Verwertungsgesellschaft - den Einsatz von technischen Schutzmaßnahmen, die das Framen von Inhalten unterbinden, zur Vorbedingung einer Lizenzierung machen können. ${ }^{17}$

Nachdem die Klage auf Vertragsabschluss ohne Auferlegung der Pflicht zur Verhinderung von Framing vom Landgericht Berlin zurückgewiesen worden war ${ }^{18}$ wurde sie auf eine Berufung hin vom Kammergericht Berlin als zulässig und begründet entschieden. ${ }^{19}$ Die von der Beklagten VG BK mit der Revision weiter verfolgte Abweisung der Klage hat der BGH dem EuGH im April 2019 mit der Frage vorgelegt, ob die Einbettung eines mit Einwilligung auf einer frei zugänglichen Internetseite verfügbaren Werkes in die Website eines Dritten im Wege des Framing überhaupt eine öffentliche Wiedergabe des Werkes im Sinne des Art. 3 Abs. 1 der Richtlinie 2001/29/EG darstellt, wenn sie unter Umgehung von durch Rechteinhaber*innen getroffenen oder veranlassten Schutzmaßnahmen gegen Framing erfolgt, ${ }^{20}$ denn eine Schutzmaßnahme, die eine rechtlich nicht relevante Nutzungshandlung unterbinden würde, kann schon aus rechtsdogmatischen Gesichtspunkten nicht als zulässige Vorbedingung für einen Lizenzvertragsabschluss einer dem Abschlusszwang unterliegenden Verwertungsgesellschaft betrachtet werden, die die von ihr wahrgenommenen Rechte auf Verlangen zu angemessenen Bedingungen einzuräumen hat. ${ }^{21}$

Die Vorlagefrage zur Auslegung des nationalen Rechts über den Umfang des Verbietungsrechtes im Hinblick auf die öffentliche Wiedergabe ist nunmehr durch den EuGH beantwortet worden.

In seinem Urteil stellt der EuGH fest, dass „Art. 3 Abs. 1 der Richtlinie 2001/29 dahin auszulegen ist, dass die Einbettung in die Website eines Dritten im Wege der Framing-Technik von urheberrechtlich geschützten und der Öffentlichkeit mit Erlaub-

14 Siehe Positionspapier „Framing leaves Artists with empty frames“: https://perma.cc/Z5FC-6 6Z6, zuletzt abgerufen am 11.05.2021.

15 Siehe Positionspapier „Closing the framing loophole“: https://perma.cc/Y6S4-2QK, zuletzt abgerufen am 11.05.2021.

16 Siehe „Joint letter on framing“: https://perma.cc/URN6-2B3D, zuletzt abgerufen am 11.05.2021.

17 Siehe zur Geschichte der Rechtsstreitigkeit auch: Euler, Digitale Bibliotheken müssen Embedding nicht verhindern, iRights Info 10.07.2018, abrufbar unter: https://doi.org/10.17176/ 20180718-145839-0.

18 Landgericht Berlin, Urt. v. 25.07.2017 - 15 O 251/16.

19 Kammergericht Berlin, Urt. v. 18.06.2018 - 24 U 146/17, abrufbar unter: https://perma.cc/A PZ3-25J7, zuletzt abgerufen am 11.05.2021.

20 BGH, Beschluss vom 25. 04.2019 - I ZR 113/18. Abrufbar unter: https://t1p.de/8jv4, zuletzt abgerufen am 11.05.2021.

21 Siehe $\$ 34$ Abs. 1 VGG. 
nis des Inhabers des Urheberrechts auf einer anderen Website frei zugänglich gemachten Werken eine öffentliche Wiedergabe im Sinne dieser Bestimmung darstellt, wenn sie unter Umgehung von Schutzmaßnahmen gegen Framing erfolgt, die der Rechtsinhaber getroffen oder veranlasst hat."

In diesem Fall werde nämlich in Ergänzung der bisherigen Rechtsprechung zur öffentlichen Wiedergabe ein neues Publikum adressiert. Nach dem EuGH ist die Wiedergabe für die gesamte Internetöffentlichkeit nur im Falle frei zugänglicher Inhalte auf frei zugänglichen Websites gegeben. Ein neues Publikum ist dagegen auch dann anzunehmen, wenn ein Dritter geschützte Werke, die mit Erlaubnis des/der Urheberrechtsinhaber*in/nen auf einer bestimmten Website frei zugänglich sind, auf eine andere Website mittels einer Link-Technologie einbindet, obwohl der/die Rechtsinhaber*in/nen technische Maßnahmen, die den Zugang zu den Werken von anderen Websites im Wege der Framing-Technologie beschränken, getroffen oder den Lizenznehmer*innen aufgegeben hat/haben, diese zu treffen, um das Publikum für die Werke allein auf die Nutzer\%innen einer bestimmten Website zu beschränken.

Daraus resultiert, dass Framing bei mit wirksamen technischen Maßnahmen geschützten Werken nur noch mit Erlaubnis der Rechteinhaber*in/nen zulässig ist, denn es stellt nach dem Verständnis des EuGH eine unautorisierte Nutzungshandlung des Dritten dar. ${ }^{22}$

Dabei berücksichtigt der EuGH ausdrücklich, dass Links im Internet, unabhängig davon, ob sie im Rahmen der Technik des Framing verwendet werden oder nicht, zum guten Funktionieren des Internets beitragen, das für die durch Art. 11 der Charta der Grundrechte der Europäischen Union gewährleistete Freiheit der Meinungsäußerung und Informationsfreiheit von besonderer Bedeutung ist, sowie zum Meinungs- und Informationsaustausch im Internet, das sich durch die Verfügbarkeit immenser Informationsmengen auszeichnet. ${ }^{23}$ Der EuGH stimmt jedoch in allen Punkten der durch die Rechteinhaber*innen vermittels ihrer Interessenvertretungen vorgetragenen Positionen zu und führt aus, dass ein Ansatz, nach dem vermutet wird, dass Rechteinhaber*innen, selbst wenn sie beschränkende Maßnahmen gegen Framing der Werke eingeführt haben, jeder Handlung der öffentlichen Wiedergabe dieser Werke durch Dritte zugunsten sämtlicher Internetnutzer zugestimmt haben, verstoßen würde gegen das ausschließliche, sich nicht erschöpfende Recht aus Art. 3 Abs. 1 und 3 der Richtlinie 2001/29, die öffentliche Wiedergabe der Werke zu erlauben oder zu verbieten.

Eine andere Beurteilung würde nach Ansicht des EuGH auf eine Erschöpfung des Rechts der Wiedergabe hinauslaufen. ${ }^{24}$

22 Siehe Pressemitteilung der die DDB rechtlich vertretenden Stiftung Preußischer Kulturbesitz vom 30.11.2021, abrufbar unter: https://perma.cc/FG4K-GXWS, zuletzt abgerufen am 11.05.2021.

23 Siehe Urteil FN 1 Rdn. 49.

24 Siehe Urteil FN 1 Rdn. 50 und 52.

RuZ 2. Jg. 3/2021 


\section{Kulturpolitische Konsequenzen}

Die Richter\%innen in Luxemburg haben erkennbar die einzelne Urheber\%in stärker in den Fokus genommen. Diese soll eine öffentliche Wiedergabe „unter Vorbehalten“ gestatten können, namentlich unter der Prämisse, dass ein Framing oder Inline-Linking durch Dritte technisch unterbunden wird. Während die einen im Urteil des EuGH eine nicht übermäßig urheberfreundliche und nicht überraschende Fortsetzung der bisherigen Rechtsprechung sehen, ${ }^{25}$ sehen andere Probleme auf die Kulturplattformen zukommen und auf deren Möglichkeit, kulturelle Inhalte im Internet zugänglich zu machen. ${ }^{26}$ Kulturplattformen wie die DDB ,geraten durch das Urteil in eine Zwickmühle. Einerseits stehen sie unter dem Erwartungsdruck, Kultur für alle sichtbar $\mathrm{zu}$ machen. Andererseits könnten sie gezwungen sein, mit technischen Barrieren zu arbeiten, um den Schutz der Urheber zu gewährleisten“. ${ }^{27}$

Vom EuGH ist der Fall zurück zum Bundesgerichtshof nach Karlsruhe gewandert, der die entscheidungsrelevanten Sachfragen im Lichte der Rechtsprechung des EuGHs zu klären hat. ${ }^{28}$ Hier wurde der Fall am 17.06.2021 im Anschluss an das EuGH Urteil erneut mündlich verhandelt (Az.: I ZR 113/18 siehe FN 20). In dieser Verhandlung deutete der Senat an, dass die Tendenz dahin gehe, dass noch weitere Sachverhaltsfeststellungen getroffen werden müssten, um am Ende in der Sache entscheiden zu können. Solche sich auf den Sachverhalt beziehenden Feststellungen kann aber nur das Instanzgericht treffen, im vorliegenden Fall also das Kammergericht in Berlin.

Voraussichtlich wird der BGH in dem für den 29. Juli 2021 angekündigtem Termin das Verfahren also zunächst nach Berlin zurückverweisen. Dort werden die Parteien Gelegenheit erhalten, zu den entscheidungsrelevanten Sachfragen nochmals vorzutragen. Bis zu einer finalen Entscheidung müssen wir uns also weiterhin gedulden, zumal die Entscheidung des Kammergerichts erneut vom BGH zu beurteilen sein könnte.

Für die Entscheidung im Hinblick auf die Sachfragen ist vorliegend festzuhalten, dass diese weitreichende Konsequenzen für den Zugang zu Kultur im Internet haben werden:

- Die Entscheidung der Sachfragen im vorliegenden Musterprozess wird europaweit richtungweisend sein und auf die Lizenzpolitik der Schwestergesellschaften der VG BK Einfluss nehmen. Es ist davon auszugehen, dass alle Verwertungsgesellschaften in Europa zukünftig den Einsatz von Schutztechnologien gegen das Framing Drit-

25 Wulff/Bardenhewer, Framing bei technisch geschützten Werken nur mit Erlaubnis des Urhebers, in: K\&R 5/2021, S. $303 \mathrm{ff}$.

26 Janisch, In der Zwickmühle, in: Süddeutsche Zeitung v. 09.03.2021, abrufbar unter: https://p erma.cc/BE86-SPYU, zuletzt abgerufen am 11.05.2021.

27 Siehe FN 20.

28 Vor allem die Frage der „Angemessenheit“ die Implementierung von wirksamen Schutzmaßnahmen i.S.v. Art. 6 InfoSoc RL 2001/29 EG gegen Framing zu verlangen gem. \34 VGG, aber auch die Frage der Vertretungsbefugnis, also der Behauptung der VG BK, im Interesse der vertretenen Rechteinhaber*innen diesen Schutz verlangen zu müssen. 
ter zur Vorbedingung der Lizenzierung machen werden, sollte die Angemessenheit der Implementierung solcher Technologien bejaht werden.

- War die Einbindung von im Internet auf frei zugänglichen Websites zugänglich gemachten Inhalten auf Websites Dritter im Wege des Framing/Embedding bislang ohne zusätzliche Lizenzierung möglich, könnte sich das abhängig von der Entscheidung zu den Sachfragen zukünftig faktisch ändern. Hierbei ist fraglich, ob die mit technischen Maßnahmen unterbundene Möglichkeit Dritter, Inhalte zu framen, im Einzelfall überhaupt erlaubt und lizenziert werden kann. Das setzte voraus, dass es technische Maßnahmen gibt, die nicht nur mit Blick auf die Inhalte differenzieren, sondern auch mit Blick auf die Nutzer\%innen, und die in der Lage sind, nur bestimmte Websites Dritte auszuschließen, es aber bestimmten Nutzer\%innen (wie z.B. Europeana oder Google) ermöglichen, die Inhalte zu framen. Die Implementierbarkeit und Kosten solcher Lösungen könnte mit Einfluss nehmen auf die Frage der Angemessenheit des Verlangens, solche Lösungen zu implementieren. Die Autorin vermutet, dass schon das Differenzieren zwischen solchen Inhalten, die vor Framing geschützt werden müssen, und solchen, die weiterhin frei zugänglich gemacht werden können, nicht ohne weiteres möglich ist. Man denke an die Abbildung eines geschützten Bildes in einem aufgeschlagenen Buch in einer Abbildung. Die Metadaten weisen Rechte nur auf der obersten Ebene und nicht für jede Abbildung der Abbildung aus und verweisen zudem regelmäßig auf die Urheber\%innen als Rechteinhaber\%innen und nicht die diese vertretenden Verwertungsgesellschaften. Ist schon die Identifikation der zu schützenden Inhalte technisch nicht möglich, bedeutet das aber, dass nur entweder alle oder keine Inhalte vor Framing Dritter geschützt werden können. Der Schutz aller Inhalte aus der DDB gegen Framing widerspräche deren Ansatz der Vernetzung, Kollaboration und auf diskursive Zusammenhänge angelegten technischen Infrastruktur der Plattform und des Internets. Die DDB könnte nicht mehr ein Hub für kulturelle Innovation sein, sondern wäre determiniert auf die Funktion einer Werbeplattform, die außerdem für diese Funktion noch Lizenzgebühren an die beworbenen Rechteinhaber*innen schuldet. Wo aggregierende Plattformbetreiber (Suchmaschinen oder Kulturplattformen) Schutzvorkehrungen nicht sinnvoll auf einzelne Inhalte anwenden können und eine pauschalisierte Anwendung auf alle Inhalte diese Freiheiten zugunsten weniger Inhalte einschränken würde, kann dies nicht im überwiegenden Interesse der Rechtehinhaber*innen sein. Insoweit ist fraglich, ob die VG BK ein repräsentatives Interesse der Mehrheit der vertretenen Rechteinhaber*innen und Mitglieder verfolgt, wenn sie diesen Schutz trotz der aufgezeigten Implikationen fordert und zur Vorbedingung einer Lizenzierung der Rechte macht.

- Die Einbindung in Kulturplattformen, welche wie Metasuchmaschinen funktionieren und auf der Technologie des Framing basieren, würde nicht mehr automatisch funktionieren. Namentlich würde das für die Kulturplattform Europeana gelten. Diese macht kulturelle Inhalte, anders als z.B. die Deutsche Digitale Bibliothek, nicht durch den Upload von Vorschaubildern zugänglich, sondern aggregiert aus- 
schließlich Metadaten, zu denen Links zu den Digitalisaten gehören, die über Viewer vermittels der Hot-Linking Methode in das europäische Portal integriert werden. Selbst wenn es technisch möglich ist, bestimmte Websites Dritter vom Schutz gegen Framing auszunehmen, würde das nunmehr eine ( $\mathrm{Zu}$-)Zahlung von Lizenzgebühren voraussetzen, damit Europeana weiterhin die gesamten Inhalte der nationalen Kulturplattformen auf einer zentralen Seite zusammenführen kann. An dieser Stelle muss angemerkt werden, dass die DDB von Anfang an die faire Entlohnung von Rechteinhaber*innen unterstützt hat und bereit war, einen Aufschlag für Europeana zu zahlen.

- Die Einbindung in Meta-Suchmaschinen wie Google, die gerade auf der Technologie des Framing basieren, würde ebenfalls nicht mehr automatisch funktionieren und damit Sichtbarkeit des Kulturerbes aus Europa massiv einschränken, was gerade das mit Europeana und DDB verfolgte Ziel der größeren Sichtbarkeit des Kulturerbes aus Europa unterminieren würde. Es ist nicht davon auszugehen, dass Google selbst bei der Möglichkeit, die technischen Schutzmaßnahmen spezifisch zu unterbinden und bestimmten Websites das Framing technisch bei Zahlung einer Lizenzgebühr zu gestatten, hierzu bereit wäre. Ausgerechnet einem kommerziellen Player wie Google das Framen kostenfrei zu gestatten, wäre hingegen kulturpolitisch bedenklich.

- Eine nicht sofort ins Auge fallende Folge könnte die Unterminierung der durch die Richtlinie über das Urheberrecht im digitalen Binnenmarkt vorgesehenen Möglichkeiten sein, nicht mehr kommerziell verfügbare Werke vereinfacht über ECL (extended collective licences) Lösungen über die Verwertungsgesellschaften zugänglich zu machen ${ }^{29}$ : Sollten diese als „repräsentativ“ angesehen werden, könnten sie verlangen die vergriffenen Werke gegen Framing durch Dritte zu schützen und damit gerade kleine Kulturerbeeinrichtungen vor eine Herausforderung bei der Umsetzung der Richtlinienziele stellen. Zudem würde die Verpflichtung von Kulturplattformen, technische Schutzmaßnahmen gegen das Framing Dritter zu implementieren, das Richtlinienziel unterminieren, die nicht kommerziell agierenden Kulturplattformen gerade aus der Problematik von TPM/DRM herauszunehmen.

- Last but not least sei die Problematik des technischen Überschutzes benannt, der darin besteht, dass Schutzmaßnahmen gegen Framing auf Seiten Dritter auch zulässige Nutzungen unterbinden würden, so z.B., wenn für Zwecke des Zitates, Memes etc. auf die Technologie des Hot- oder Inline-Linking zurückgegriffen wird.

\section{Ableitungen und offene Fragen}

Wenn eine öffentliche Wiedergabe im Falle des Framing erst in der Umgehung technischer Schutzmaßnahmen gegen Framing vorliegt, bedeutet das im Umkehrschluss, dass

29 Art. 8 RL EU 2019/790, dessen Umsetzung vorgesehen ist durch $\$ 61 d$ UrhG-E i.V.m $\iint 52 b$ VGG-E. 
dieses als Grundprinzip (per Default) erst einmal zulässig bleibt und keine öffentliche Wiedergabe darstellt, die der Erlaubnis bedarf. Rechteinhaber*innen müssen erst tätig werden und Schutzmaßnahmen gegen das Framing vorsehen bzw. diese veranlassen, wenn sie dieses Prinzip durchbrechen wollen. Eine rechtliche Wertung, die Framing qua definitionem grundsätzlich als Nutzungshandlung festlegt, wie sie sich die VG BK durch Korrektur der bisherigen Rechtsprechung erhofft hat, ist durch das aktuelle Urteil des EuGH nicht erfolgt. Dabei wollte die VG BK nie Kulturerbeeinrichtungen mit Framingverhinderungstechnologien überfordern, sondern hat selbst damit argumentiert, dass das Framing deswegen in den Anwendungsbereich der Verbietungsrechte aufgenommen werden müsste, ohne dass dies eine Handlung auf Seiten der Rechteinhaber*innen erforderlich macht, da das wiederum die Kulturerbeeinrichtungen vor schwierige Herausforderungen stellen würde. Deutlich wird das schon in einer gemeinsam von VG BK und Kulturerbeeinrichtungen formulierten Position: In der sogenannten Münchner Note $^{30}$ fordern die Kulturerbeeinrichtungen gemeinsam mit der VG BK als Lösung für das Problem eines bilderlosen Europas eine vergütungspflichtige Schrankenbestimmung für die Visualisierung der kulturellen Schätze, sowie daneben eine Regulierung des Framing. Die Notwendigkeit für die Regulierung des Framing wird damit begründet, dass, solange Framing nicht einem gesetzlichen Verbotsrecht unterliege, geschützte Werke nur eingeschränkt in zugangsbeschränkenden Internetangeboten gezeigt werden könnten, ${ }^{31}$ oder aber nur, sofern durch Technologien geschützt, die das Framing verhindern (Code as Law). ${ }^{32}$

Die VG BK sieht in der Einordnung des Framing als urheberrechtliche Nutzungshandlung die einzige Alternative dazu, dass Kulturerbeeinrichtungen geschützte Werke

30 Münchner Note „Museen, Bibliotheken und Archive fordern dringend notwendige politische Unterstützung zur Sichtbarmachung ihrer Sammlungsbestände im digitalen Raum“. 2018, abrufbar unter: https://perma.cc/F6JB-8QCS, zuletzt abgerufen am 11.05.2021.

31 Siehe nur „Framing muss ins Brüsseler Paket“: https://web.archive.org/web/20161130223 040/http://www.bildkunst.de/urheberrecht/die-hyperlink-problematik/framing-muss-ins-b ruesseler-urheberrechts-paket.html, zuletzt abgerufen am 11.05.2021; „EuGH enteignet Urheber": https://web.archive.org/web/20161130170339/http://www.bildkunst.de/urheber recht/die-hyperlink-problematik/eugh-enteignet-bildurheber.html, zuletzt abgerufen am 11.05.2021; Position VG zur UrhReform EU: https://web.archive.org/web/2016113022 3216/http://www.bildkunst.de/urheberrecht/position-zur-geplanten-eu-reform-2016.html, zuletzt abgerufen am 11.05.2021; sowie zuletzt die formulierte Forderung an den EuGH klarzustellen, dass sich die Zustimmung zur Nutzung eines Werkes im Internet immer nur auf die konkrete Webseite bezieht und die Einbettung des Werkes in weiteren Webseiten nicht von einer solchen Zustimmung erfasst ist: https://perma.cc/T3LR-GUGC, zuletzt abgerufen am 11.05.2021.

32 „Framingverhinderungstechnologie“ basiert zum Beispiel darauf, Bilder nur mit einer dynamischen, statt mit einer stabilen Webadresse zu versehen. Den Kern von Lösungen, die das Framing von Bilddateien technisch verhindern, bilden kryptografische Verfahren. Verschlüsselt werden dabei nicht die Bilder selbst. Stattdessen werden die Links auf die Bilddateien so manipuliert, dass diese nur einmalig bzw. für einen begrenzten Zeitraum abgerufen werden können und es für Dritte nicht einfach möglich ist, aus einem solchen temporären Link auf ein Bild einen (gültigen) anderen Link zu erzeugen, der zur Einbindung des betreffenden Bildes in eine andere Webseite genutzt werden kann. Dieses Verfahren ist auch ohne Verschlüsselung umsetzbar. 
aus dem VG BK-Repertoire bei einer Zugänglichmachung vor dem Framing Dritter schützen, da diese damit überfordert seien, sodass im Ergebnis eine Lösung für das Zeigen von Werken der bildenden Kunst als Bestandteil des kulturellen Erbes durch Kulturerbeeinrichtungen auf vertraglicher Ebene ausscheide. ${ }^{33}$ Weil also faktische Kontrolle durch den Schutz vor Framing hier nicht herstellbar sei, sondern im Falle der Online-Sammlungen die Kulturerbeeinrichtungen überfordert seien, brauche es im Ergebnis eine gesetzliche Regulierung des Framing, das per default als Nutzungshandlung zu bewerten sei.

Die VG BK sieht die EuGH-Entscheidung daher aus den folgenden Gründen zwiespältig: „Einerseits hat der EuGH Künstler*innen die Kontrolle über die Nutzung ihrer Werke im Internet zurückgegeben und seine Rechtsprechung geschärft, andererseits sehen wir auch, dass es für die Lizenznehmer*innen eine Bürde ist, wenn immer eine technische Sicherung erfolgen muss. Wir hätten uns gewünscht, dass der EuGH weiter geht und auch für das Internet festhält, was in der analogen Welt Standard ist: dass eine Lizenz immer nur den oder die Lizenznehmer* in zur Nutzung berechtigt, nicht aber jede*n beliebige*n Dritte*n. “34

Was hier aufeinanderprallt, ist einerseits das Netz als dezentrale und nicht kontrollierte Form der Wissensteilung, in der Werke qualifizierte Kommunikationen sind, die permanent zum Gegenstand von Anschlusskommunikationen gemacht werden, andererseits das Wirtschaftssystem, in dem die Kommunikationen Gegenstand von Handelstransaktionen sind, sofern sie auf einem Markt gehandelt werden können und in dem um den Marktmechanismus zu ermöglichen Zugangsbeschränkungen und Ausschließlichkeitsrechte errichtet werden. ${ }^{35}$

Jedoch haben sich die Kulturerbeeinrichtungen, die gemeinsam mit der VG BK die Münchner Note unterzeichnet haben, mit Blick auf die urheberrechtspolitische Forderung nach Regulierung des Framing unnötig vor den (fremden) Karren der Rechteinhaber*innen spannen lassen. ${ }^{36}$ Der Direktor des Marta Museums in Herford, der für diese Institution die Münchner Note unterzeichnet hat, ruft mittlerweile unter der Überschrift „Kontrolle ist gut - Freiheit ist besser“ zum teilweisen Rücktritt auf und erklärt in einem Blogbeitrag: „Als Museum für zeitgenössische Kunst plädieren wir nachdrücklich für eine Sicherung des Bildungsauftrags der Museen, Bibliotheken

33 Die Technologien, mit denen Framing verhindert werden kann, sind für Sachverhalte gedacht, bei denen die gesamte Website oder einzelne Inhalte gegen Framing geschützt werden sollen und lassen sich nicht einfach übertragen auf aggregierende Plattformen, bei denen vom Gesamtangebot weniger als 1 Prozent der Inhalte betroffen sind, die sich im Einzelnen nicht auffinden lassen, weil die Metadaten z.B. ein geschütztes Bild als Teil einer Abbildung auf einer Seite in einem mehrseitigen Buch nicht separat ausweisen können.

34 Siehe Anke Schierholz, Justiziarin der VG BK. Zitiert auf der Website der Initiative Urheberrecht am 09.03.2021, abrufbar unter: https://perma.cc/6ZRL-2YB2, zuletzt abgerufen am 11.05.2021.

35 Ausgeführt bei Grünberger, Zugangsregeln bei Verlinkungen auf rechtswidrig zugänglich gemachte Werke, in: ZUM 2016, S. 906.

36 Nachtigäller, Direktor des Marta Herford, siehe: https://perma.cc/9RWB-EP7X, zuletzt abgerufen am 11.05.2021. 
und Archive, für einen beidseitig fairen (!) Ausgleich zwischen den Interessen der Urheber*innen und dem gesellschaftlichen Zugang zu den Schöpfungen. Wir identifizieren uns ungebrochen mit der Intention der Münchner Note, allerdings ohne den Framing-Passus. Ich und wir sprechen uns gegen die juristische Kontrolle über die Weiterverwendung von Werken in diskursiven Zusammenhängen aus. Forschungsfreiheit, Diskussionskultur und umfassende Dokumentation sind ein hohes Gut unserer Gesellschaft.“

Ein Zustand der grundsätzlichen Erlaubnispflichtigkeit des Framing würde zwar das Wirtschaftssystem stärken, aber das Kommunikationssystem stark beschränken und kann schon deswegen nicht gesetzlich festgeschrieben werden, da das im Ergebnis dazu führen würde, dass nur noch die Inhalte in der Logik des Internets verwendbar wären, bei denen aktiv hierfür Sorge getragen wurde. Im Spannungsfeld „Open Access und wissenschaftliche Information" sehen wir, dass bestenfalls 30 Prozent der Rechteinhaber*innen von dieser Möglichkeit Gebrauch machen und durch Nutzung freier Lizenzen das Regel-Ausnahme-Verhältnis von Freiheit und Zugangsbeschränkung wiederherstellen. Zudem ist davon auszugehen, dass nur wenige Rechteinhaber*innen ein Interesse an der Unterbindung der Möglichkeit von Framing haben. Die Implementierung von technischen Schutzmaßnahmen gegen das Framing hat nämlich wie aufgezeigt weitreichende, negative Auswirkungen für alle Beteiligten.

Auch und obwohl das Urheberrecht als ordnungspolitisches Regelungsinstrumentarium eigentlich kein Kontrollinstrument sein soll, sondern sein unbestrittener Regelungszweck in der angemessenen Beteiligung der Werkurheber*innen an der Verwertung „ihrer" Werke liegt und aus kultureller Sicht vor allem die Aufrechterhaltung eines fortwährenden Schöpfungskreislaufs im Vordergrund steht, ${ }^{37}$ lässt es sich dennoch im digitalen Umfeld genau hierzu potentiell einsetzen, da hier bis auf wenige Ausnahmen (wie das Framing) jede Aktivierung des Inhalts zugleich auch in den meisten Fällen eine urheberrechtlich relevante Nutzungshandlung bedeutet. ${ }^{38}$ Dem Urheberrecht kommt damit im digitalen Raum eine entscheidende Bedeutung für die Kontrolle der Kommunikation und die Weitergabe und Vermittlung kultureller Äußerungen und Inhalte $\mathrm{zu}^{39}$

Die Grundrechte verpflichten dazu, beiden Handlungslogiken - Zugangsfreiheit und Zugangskontrolle - gleichzeitig gerecht zu werden. ${ }^{40}$ Die Grundrechte verpflichten das Urheberrecht im Ergebnis darauf, angemessene Lösungen für die systemspezifische Abstimmung von Ausschließlichkeit und Nutzungsfreiheit zu finden. Dieser angemessene Ausgleich erfordert, dass das Recht neben Instituten der Zugangsbeschrän-

37 Senftleben, Der kulturelle Imperativ des Urheberrecht, in: Weller/Kemle/Dreier, Kunst im Markt - Kunst im Recht, $75 \mathrm{ff}$.

38 Zumindest immer eine Vervielfältigung gem. $\$ 16$ UrhG, die nur als vorübergehende und technisch notwendige Vervielfältigung auch gem. $\ 44$ a UrhG privilegiert ist.

39 Vgl. Peukert, GRUR-Beilage 2014, S. 77.

40 Wielsch, ZGE2013, S. 299ff.

RuZ 2. Jg. 3/2021 
kung (subjektiven Rechten) auch spezifische Institute zur Vermittlung und Förderung von freien Nutzungsmöglichkeiten bereitstellt. ${ }^{41}$

Der visuelle Nachweis von Werken ist gerade für die Museen von hervorgehobener Bedeutung. Während es für den analogen Bereich eine privilegierende Schrankenbestimmung gibt, nämlich die mit Inkrafttreten des UrhWissG vergütungspflichtig gewordene Katalogbildschranke aus $\mathbb{S}$ 60e III, 60f I UrhG, existiert eine solche Privilegierung für den digitalen Raum nicht. Den entsprechenden Bedarf haben die Kulturerbeeinrichtungen über den Museumsbund schon 2012 in einem Positionspapier formuliert. ${ }^{42}$

$\mathrm{Ob}$ die Lizenzierung von der Verpflichtung des Einsatzes von Framingverhinderungstechnologien abhängig gemacht werden kann, ist als offene Sachfrage noch durch den BGH abschließend zu beantworten. Dieser darf die Entscheidung der Sachfragen im vorliegenden Musterverfahren in Übereinstimmung mit der Grundregel, dass zeitlich beschränkte Ausschließlichkeitsrechte die Ausnahme darstellen, während Freiheit der Kommunikation die Regel darstellt, ${ }^{43}$ und der Rechtsprechung des EuGH nicht nur auf eine vorrangig technologisch, funktionale Betrachtung stützen, sondern muss ebenso mögliche Implikationen im Hinblick auf sozialnützliche Funktionen des Internets und die Gewährleistung der Informations- und Meinungsfreiheit im Internet durch Suchmaschinen berücksichtigen. ${ }^{44}$

Mit Blick auf die dargestellten weitreichenden negativen Implikationen darf bezweifelt werden, ob die Anwendung wirksamer (!) technischer Schutzmaßnahmen gegen das Framing Dritter eine angemessene Vorbedingung für die Lizenzierung darstellt. Anders, als vielfach von den verschiedenen Interessenvertretern behauptet, ist verantwortlich für das Fehlen von zeitgenössischen Werken der bildenden Kunst aus Europa im Internet allein die mangelnde Bereitschaft der Verwertungsgesellschaften, die Zugänglichmachung von Abbildungen Werken aus ihrem Repertoire ohne die Pflicht zur Implementierung von technischen Schutzmaßnahmen, die das Framing Dritter verhindern, zu lizenzieren. Nicht hingegen die Rechtsprechung des EuGH bzw. der vom europäischen Urheberrechtsgesetzgeber gesteckte Rahmen, innerhalb dessen eine solche Lizenzierung umsetzbar wäre. Wir riskieren im Ergebnis ein bilderloses Europa nur deswegen, weil praktikable Lizenzierungslösungen trotz Vorliegens aller Voraussetzungen nicht umgesetzt werden. Statt sich auf einen möglichen Missbrauch zu konzentrieren, sollte die positive Nutzung im Vordergrund der Suche nach sachgemäßen Lösungen stehen. Die aktuelle Situation ist eine Lose-Lose-Situation, denn weder profitieren die Rechteinhaber*innen, noch die Allgemeinheit, die keinen Zugang zu zeitgenössischen Abbildungen von Werken der bildenden Kunst als Teil des digital

41 Wielsch, Zugangsregeln 2008, S. 42.

42 Kulturelles Erbe im Internet sichtbar machen. Positionspapier Deutscher Museumsbund 2012; in: Klimpel/Euler (Hrsg.), Der Vergangenheit eine Zukunft 2015, 292, abrufbar unter: https://doi.org/10.17176/20180716-114912-0.

43 Peukert, Gemeinfreiheit - Begriff, Funktion, Dogmatik 2012, RN 65.

44 BGH MMR 2010, 475, Vorschaubilder I, sowie BGH ZUM 2018, 123 Rn. 60 ff. - Vorschaubilder III 
verfügbaren Kulturerbes hat. ${ }^{45}$ Die Annahme ist noch nicht überprüft worden, dass dann keine sinnvollen Lizenzierungsszenarien mehr denkbar sind, wenn das Framing nicht mit wirksamen technischen Maßnahmen unterbunden wird, weil in allen anderen Fällen der Nutzung davon auszugehen sei, dass Nutzer die Inhalte framen würden. Die unmittelbaren negativen Folgen einer umfassend eingesetzten Framingverhinderungstechnologie sind jedoch offensichtlich. Zugleich ist der behauptete Lizenzschaden nicht evidenzbasiert. Ein tatsächlicher Schaden bestünde in dem Betrag, der aufgrund veränderter Situation nicht mehr erwirtschaftet wird. Dagegen nicht in dem Betrag, der bei Verhinderung des Framing zusätzlich eingetrieben werden könnte. Zählt man die Nutzungen durch Framing, kommt man zwar zu atemberaubenden Zahlen, diese geben aber nicht realistisch wieder, was tatsächlich lizenziert werden würde. ${ }^{46} \mathrm{Im}$ Gegenteil ist davon auszugehen, dass die übermäßige Unterbindung der Möglichkeit, zu framen, zu einer Reduktion des (lizenzierten) Framing weniger erfolgreicher Inhalte beitragen würde, aber in den allermeisten anderen Fällen der Aufwand der Rechteklärung und damit verbundenen Transaktionskosten nicht betrieben werden würde. ${ }^{47}$

Bei 100 Prozent Werken kommt schätzungsweise nur ca. 2-4 Prozent überhaupt ein kommerzieller Wert in dem Sinne zu, dass sie nachgefragt werden. ${ }^{48}$ Das zeigt auch vorliegender Fall, in dem Kulturerbeeinrichtungen die Sichtbarmachung verborgener Kulturschätze anstreben, die in über 95 Prozent der Fälle (leider) kaum einmal aufgerufen, geschweige denn geframt werden. Ob nicht das Interesse an Sichtbarkeit der 95 Prozent der Rechteinhaber*innen das kommerzielle Interesse der übrigen 5 Prozent überwiegt, ist ebenso zu ermitteln, wie die Angemessenheit der Lizenzhöhe, auf die sich die Frage zuzuspitzen droht und die nicht auf eine Schutzgelderpressung hinauslaufen darf.

Ansonsten könnte es sein, dass sich DDB, andere nationale Kulturplattformen und Europeana im Ergebnis eben doch auf gemeinfreies Material beschränken müssten. Das ist dann jedoch mit Sicherheit nicht die gewünschte Lösung. Für dieses Ergebnis hätte man sich alle Verhandlungen mit der VG BK und das Gerichtsverfahren sparen können.

\section{(9) (1) ( ๑) $\odot$ Ellen Euler}

45 Vgl. Gespräch über die Zukunft des Urheberrechts, in dem Künstlerinnen eindeutig zu verstehen geben, dass sie auf Sichtbarkeit mehr Wert legen, als auf Lizenzgebühren, siehe: https: //perma.cc/Y5UY-JFC9, zuletzt abgerufen am 11.05.2021 sowie Euler, C\&R 2013, 616 f.

46 So aber Hanfeld unter Berufung auf den Bundesverband professioneller Bildanbieter (bvpa), FAZ Feuilleton vom 14.07.2018, abrufbar unter: https://perma.cc/9K8B-Y4LJ, zuletzt abgerufen am 11.05.2021.

47 Siehe Conrad, Kuck mal wer da spricht, in: CR 2013, 305. Verfügbar bei SSRN: https://paper s.ssrn.com/sol3/papers.cfm?abstract_id=2249879, zuletzt abgerufen am 11.05.2021, hier S. $15 \mathrm{f}$.

48 Vgl. Frentz zitiert nach Kohle, Museen digital 2018, S. 31. 